\title{
Taraxacum coreanum protects against glutamate-induced neurotoxicity through heme oxygenase-1 expression in mouse hippocampal HT22 cells
}

\author{
CHI-SU YOON $^{1 *}$, WONMIN KO ${ }^{1 *}$, DONG-SUNG LEE ${ }^{2}$, DONG-CHEOL KIM ${ }^{1}$, JONGSU KIM ${ }^{1}$, \\ MOONBUM CHOI ${ }^{1}$, JIN SEON BEOM ${ }^{1}$, REN-BO AN $^{3}$, HYUNCHEOL OH $^{1}$ and YOUN-CHUL KIM ${ }^{1,3}$ \\ ${ }^{1}$ College of Pharmacy, Wonkwang University, Iksan, Jeonbuk 54538; ${ }^{2}$ Department of Biomedical Chemistry, \\ College of Health and Biomedical Science, Konkuk University, Chungju, Chungbuk 27478, Republic of Korea; \\ ${ }^{3}$ Key Laboratory of Natural Resources of Changbai Mountain \& Functional Molecules (Yanbian University), \\ Ministry of Education, Yanji, Jilin 133002, P.R. China
}

Received December 22, 2015; Accepted January 5, 2017

DOI: $10.3892 / \mathrm{mmr} .2017 .6237$

\begin{abstract}
Taraxacum coreanum Nakai is a dandelion that is native to Korea, and is widely used as an edible and medicinal herb. The present study revealed the neuroprotective effect of this plant against glutamate-induced oxidative stress in HT22 murine hippocampal neuronal cells. Ethanolic extracts from the aerial (TCAE) and the root parts (TCRE) of T. coreanum were prepared. Both extracts were demonstrated, by high performance liquid chromatography, to contain caffeic acid and ferulic acid as representative constituents. TCAE and TCRE significantly increased cell viability against glutamateinduced oxidative stress in mouse hippocampal HT22 cells. Western blot analysis revealed that treatment of HT22 cells with the extracts induced increased expression of the enzyme heme oxygenase-1 (HO-1), compared with untreated cells, in a concentration-dependent manner. Increased HO-1 enzymatic activity, compared with untreated cells, was also demonstrated following treatment with TCAE and TCRE. In addition, western blot analysis of the nuclear fractions of both TCAE and TCRE-treated HT22 cells revealed increased levels of nuclear factor erythroid 2 like 2 (Nrf2) compared with untreated cells, and decreased Nrf2 levels in the cytoplasmic fraction compared with untreated cells. The present study suggested that the neuroprotective effect of T. coreanum is associated with induction of HO-1 expression and Nrf2 translocation to the nucleus. Therefore, $T$. coreanum exhibits a promising
\end{abstract}

Correspondence to: Professor Youn-Chul Kim, College of Pharmacy, Wonkwang University, 460 Iksan Boulevard, Iksan, Jeonbuk 54538, Republic of Korea

E-mail: yckim@wku.ac.kr

*Contributed equally

Key words: Asteraceae, glutamate, heme oxygenase-1, HT22 cells, neuroprotection, Taraxacum coreanum function in prevention of neurodegeneration. Further studies will be required for the isolation and the full characterization of its active substances.

\section{Introduction}

Oxidative stress is involved in the progress of neuronal degenerative disorders, such as multiple sclerosis (1). Glutamate is a crucial neurotransmitter for neural activation. However, when released as a result of neural injury, high concentrations of extracellular glutamate are toxic to neurons. Glutamate cytotoxicity is a result of non-receptor-mediated oxidative stress and receptor-initiated excitotoxicity (2). Mouse hippocampal neuronal HT22 cells have previously been used to examine the mechanism of glutamate-induced oxidative stress (3); because HT22 cells express low levels of glutamate receptors, glutamate-mediated cell damage in these cells is primarily due to non-receptor-mediated oxidative stress $(4,5)$.

The rate limiting enzyme in heme catabolism is heme oxygenase-1 (HO-1; official gene symbol HMOX1), which is thus a crucial component in the cellular antioxidant system. HO-1 gene expression is inducible (6) and HO-1 has been demonstrated to be involved in cytoprotection against glutamate-induced oxidative damage in HT22 cells (7). Induction of HO-1 expression is performed at the transcriptional level, and its expression is regulated by nuclear factor erythroid 2 like 2 (Nrf2; official gene symbol Nfe2l2) (8). Nrf2 has been reported to induce expression of various antioxidant stressrelated proteins, including glutathione (GSH) and HO-1 (9).

Taraxacum coreanum Nakai (Asteraceae family) is a dandelion native to Korea, and it is extensively consumed as a vegetable and used as a traditional therapeutic agent for inflammatory diseases in Korea. Previous studies of this species have revealed the presence of taraxinic acid-1'-O- $\beta$ D-glucopyranoside, of which the hydrolysate, taraxinic acid, possesses an anti-leukemic effect (10). The anti-inflammatory effect of a methanolic extract of the aerial part of T. coreanum has also been reported (11). In the present study, which aimed to investigate the neuroprotective potential of natural Korean 
medicinal sources, the cytoprotective effect of $T$. coreanum against oxidative stress was evaluated in vitro in HT22 cells.

\section{Materials and methods}

Preparation of plant extract. The whole plant of Taraxacum coreanum Nakai was collected from the Botanical Garden of Wonkwang University (Iksan, Korea) in May 2014. This species was identified by Dr Kyu-Kwan Chang, and the voucher specimen (WK-2014-028) was deposited in Wonkwang University. Each fresh aerial and root part (50 g) was soaked in $500 \mathrm{ml}$ ethanol and left for 7 days at room temperature. Following filtration with filter paper, the solvent was dried using rotary evaporator to yield $7.5 \mathrm{~g}$ of aerial ethanolic extract (TCAE) and $12.8 \mathrm{~g}$ of root ethanolic extract (TCRE).

High performance liquid chromatography (HPLC). Chromatography was performed using a HPLC instrument of the YL-9100 series (YoungLin Instrument Co., Ltd., Anyang, Korea). In all experiments, a Capcell Pak C18 column (4.6x250 mm, $5 \mu \mathrm{m}$; Shiseido Co., Ltd, Tokyo, Japan) was used as the stationary phase, and the injection volume was $20 \mu 1$. Samples containing $2 \mathrm{mg} / \mathrm{ml}$ of TCAE or TCRE were prepared. The mobile phase consisted of water containing $0.1 \%$ formic acid (A) and acetonitrile (B) in a gradient system: 0-50 min linearly changed 10 to $50 \%$ B, 50-55 min linearly changed 50 to $100 \%$ $\mathrm{B}, 55-60$ min remained at $100 \% \mathrm{~B}$. The detection wavelength was adjusted to $254 \mathrm{~nm}$, and the flow rate was $0.7 \mathrm{ml} / \mathrm{min}$.

Chemicals and reagents. All cell culture reagents were obtained from Gibco; Thermo Fisher Scientific, Inc. (Waltham, MA, USA). Tin protoporphyrin (SnPP) and cobalt protoporphyrin IX (CoPP) were obtained from Frontier Scientific, Inc. (Logan, UT, USA). Caffeic acid, chlorogenic acid, ferulic acid, and all other chemicals, unless indicated otherwise, were obtained from Sigma-Aldrich; Merck Millipore (Darmstadt, Germany).

Cell culture and viability assay. Mouse hippocampal HT22 cells were obtained from Professor Hyun Park (Wonkwang University). Cell culture and MTT assay were conducted as described previously (12). Briefly, a total of $2 \times 10^{4}$ cells/well were seeded in 96-well plates. They were pre-treated with the indicated concentration of TCAE or TCRE for $3 \mathrm{~h}$, and this was followed by treatment with $5 \mathrm{mM}$ glutamate. For measurement of cell viability, cells were maintained with 3-(4,5-dimethylthiazol-2-yl)-2,5-diphenyltetrazolium bromide (MTT) at a final concentration of $0.5 \mathrm{mg} / \mathrm{ml}$ for $4 \mathrm{~h}$, and the formazan formed was dissolved in acidic 2-propanol.

Western blot analysis. HT22 cells were harvested and pelleted by centrifugation at $200 \mathrm{x} g$ for $3 \mathrm{~min}$. Subsequently, the cells were rinsed with PBS and lysed using radioimmunoprecipitation assay (RIPA) lysis buffer containing $25 \mathrm{mmol} / \mathrm{l}$ Tris- $\mathrm{HCl}$ buffer (pH 7.6), $150 \mathrm{mmol} / \mathrm{l} \mathrm{NaCl}, 1 \%$ NP-40, $1 \%$ sodium deoxycholate, and $0.1 \%$ SDS for $15 \mathrm{~min}$ at $4^{\circ} \mathrm{C}$, and then underwent centrifugation at $15,000 \times \mathrm{g}$ for $10 \mathrm{~min}$ at $4^{\circ} \mathrm{C}$. The protein concentration was determined using Bradford Assay Reagent (Bio-Rad Laboratories, Inc., Hercules, CA, USA). A total of $30 \mu \mathrm{g}$ protein samples were resolved using SDS-polyacrylamide gel electrophoresis and electrophoretically transferred onto a nitrocellulose membrane. The membrane was blocked with 5\% skimmed milk, washed with TBST buffer, and then incubated with the following primary antibodies: Anti-HO-1 (catalog no. sc-10789; 1:1,000), anti-Nrf2, (catalog no. sc-722; 1:1,000) antiLamin B, (catalog no. sc-6216; 1:1,000), anti-Actin (catalog no. sc-1616; 1:1,000) all from Santa Cruz Biotechnology, Inc. (Dallas, TX, USA). They were then incubated with horseradish peroxidase-conjugated goat (catalog no. ap106p; 1:1,000) and rabbit (catalog no. ap132p; 1:1,000) secondary antibodies, obtained from EMD Millipore (Billerica, MA, USA), followed by ECL detection. Primary and secondary antibodies were diluted with $3 \%$ skimmed milk in TBST buffer. The bands were visualized with enhanced chemiluminescence (GE Healthcare Life Sciences, Chalfont, UK) and quantified by densitometry (Image $\mathbf{J}$, National Institutes of Health, USA). Nuclear and cytoplasmic extracts of cells were prepared using NE-PER reagents, as per the manufacturer's instructions (Thermo Fisher Scientific, Inc.).

HO-1 activity. Determination of $\mathrm{HO}$ activity occurred as previously described by Motterlini et al (13). Briefly, the HT22 cells were scraped off the dish, and centrifuged $\left(1,000 \times g\right.$ for $10 \mathrm{~min}$ at $\left.4^{\circ} \mathrm{C}\right)$. The pellet of HT22 cell was suspended in $\mathrm{MgCl}_{2}$ phosphate buffer (20 mM, pH 7.4), frozen at $-70^{\circ} \mathrm{C}$, and finally sonicated on ice prior to centrifugation at $18,000 \times g$ for $10 \mathrm{~min}$ at $4^{\circ} \mathrm{C}$. The supernatant was added to a nicotinamide adenine dinucleotide phosphate (NADPH)-generating system containing $0.8 \mathrm{mM}$ NADPH, $2 \mathrm{mM}$ glucose-6-phosphate, $0.2 \mathrm{U}$ glucose-6-phosphate- ${ }_{-}{ }^{-}$ dehydrogenase, and $2 \mathrm{mg}$ protein of rat liver cytosol prepared from the $15,000 \times \mathrm{g}$ supernatant fraction as a source of biliverdin reductase, potassium phosphate buffer $(100 \mathrm{mM}$, $\mathrm{pH}$ 7.4), and hemin $(10 \mu \mathrm{M})$ in a final volume of $200 \mu 1$. The reaction was conducted for $1 \mathrm{~h}$ at $37^{\circ} \mathrm{C}$ in the dark and terminated by addition of $1 \mathrm{ml}$ chloroform. The extracted bilirubin was calculated by the difference in absorption between wavelengths of 464 and $530 \mathrm{~nm}$ using a quartz cuvette (extinction coefficient, $40 \mathrm{mM}^{-1} \mathrm{~cm}^{-1}$ for bilirubin).

Preparation of cytosolic and nuclear fractions. HT22 Cells were homogenized (1:20,w:v) in PER-Mammalian Protein Extraction buffer (Pierce; Thermo Fisher Scientific, Inc.) including freshly added $1 \mathrm{mM}$ phenylmethylsulfonyl fluoride and protease inhibitor cocktail I (EMD Millipore). The cytosolic fraction of the cell was prepared by centrifugation at $15,000 \times \mathrm{g}$ for $10 \mathrm{~min}$ at $4^{\circ} \mathrm{C}$. Cytoplasmic and nuclear extracts of the cells were prepared using NE-PER cytoplasmic and nuclear extraction reagents (Pierce; Thermo Fisher Scientific Inc.), respectively. These methods were conducted as previously described (12).

Statistical analysis. Data were expressed as the mean \pm standard deviation of at least 3 independent experiments. To compare 3 or more groups, one-way analysis of variance followed by the Newman-Keuls post hoc test was used. Statistical analysis was performed using GraphPad Prism software version 3.03 (GraphPad Software Inc., La Jolla, CA, USA). $\mathrm{P}<0.05$ was considered to indicate a statistically significant difference. 

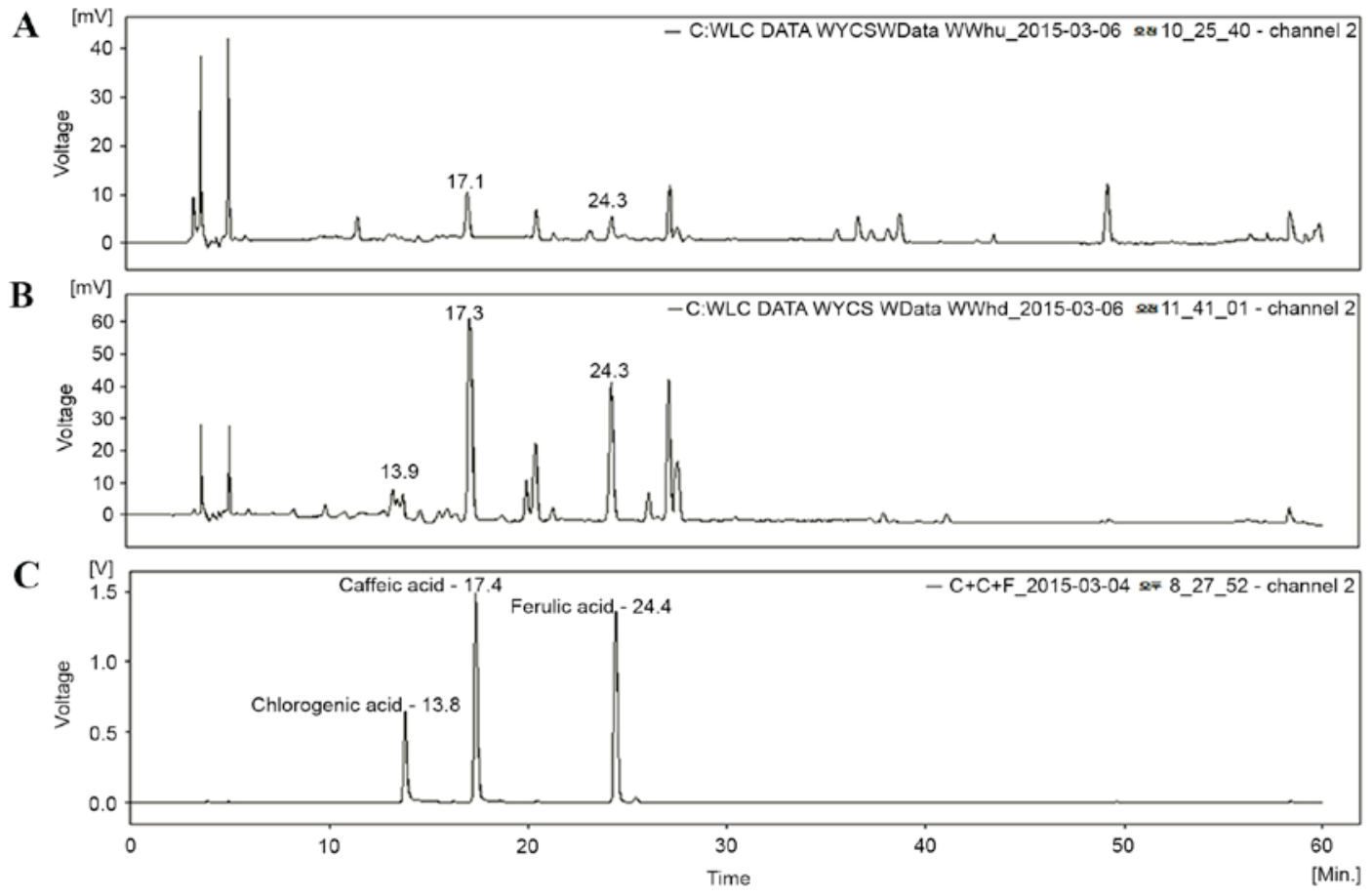

Figure 1. High performance liquid chromatography chromatograms of (A) TCAE, (B) TCRE and (C) internal standard compounds. TCAE, Taraxacum coreanum aerial extract; TCRE, T. coreanum root extract.

\section{Results}

HPLC analysis of TCAE and TCRE. Three phenolic compounds, ferulic acid, caffeic acid and chlorogenic acid, have been isolated from plants of the Taraxacum genus $(14,15)$. Therefore, TCAE and TCRE were analyzed by HPLC in order to assess the presence of these compounds. As demonstrated in Fig. 1, the peaks of ferulic acid and caffeic acid appeared clearly in the HPLC chromatograms of both TCAE (Fig. 1A) and TCRE (Fig. 1B), however, chlorogenic acid was only detected in TCRE (Fig. 1B).

Effects of TCAE and TCRE on oxidative toxicity. To investigate the protective effects of TCAE and TCRE against glutamate-induced cytotoxicity, HT22 cell viability was measured by MTT assay. Non-cytotoxic effects were obtained with concentrations of up to $400 \mu \mathrm{g} / \mathrm{ml}$ for both TCAE and TCRE (data not shown). The viability of glutamate-treated HT22 cells $(5 \mathrm{mM}$ for $24 \mathrm{~h}$ ) was tested following treatment with $0,50,100,200$ and $400 \mu \mathrm{g} / \mathrm{ml}$ of each extract (Fig. 2). The results demonstrated that both TCAE and TCRE significantly restored the cell viability following glutamate-mediated damage in a concentration-dependent manner compared with cells treated with glutamate only (Fig. 2). Trolox, a wellknown antioxidant, was used as a positive control in this assay and was confirmed to exhibit a significant protective effect (Fig. 2). The present results suggested that both aerial and root ethanolic extracts of $T$. coreanum could protect HT22 cells against oxidative stress.

Effects of TCAE and TCRE on HO-1 expression and activity. The effect of TCAE and TCRE on HO-1 expression was examined in HT22 cells. When cells were treated with

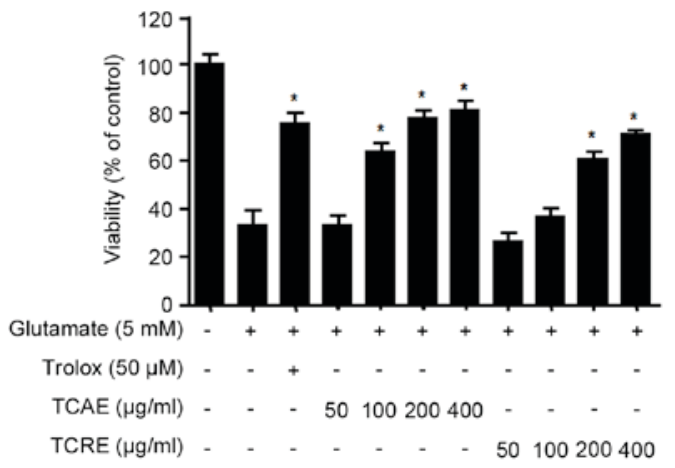

Figure 2. Effects of TCAE and TCRE on glutamate-induced oxidative toxicity in HT22 cells. The cells were incubated with either $50 \mu \mathrm{M}$ Trolox (as a positive control) or 50,100, 200 or $400 \mu \mathrm{g} / \mathrm{ml}$ TCAE or TCRE (for $3 \mathrm{~h}$ ). Cell damage was then induced by treatment with $5 \mathrm{mM}$ glutamate in HT2 2 cells for $12 \mathrm{~h}$. Viability was then measured by MTT assay. ${ }^{*} \mathrm{P}<0.05$ vs. glutamate-treated cells. TCAE, Taraxacum coreanum aerial extract; TCRE, T. coreanum root extract.

$50,100,200$ or $400 \mu \mathrm{g} / \mathrm{ml}$ TCAE or TCRE for $12 \mathrm{~h}$, both extracts visibly increased the expression of $\mathrm{HO}-1$ protein in a concentration-dependent manner, compared with untreated cells (Fig. 3A). In agreement with the concentration-dependent expression of HO-1, both TCAE and TCRE treatments also significantly increased HO-1 activity in HT22 cells compared with untreated cells (Fig. 3B). CoPP, used as a positive control, exhibited a prominent induction of both HO-1 protein expression and activity (Fig. 3).

Effects of HO-1 inhibitor on the cytoprotection activity of TCAE and TCRE. The hypothesis that the protective effect of TCAE and TCRE in HT22 cells arose from HO-1 expression was further tested. Pretreatment of cells with SnPP, a 
A
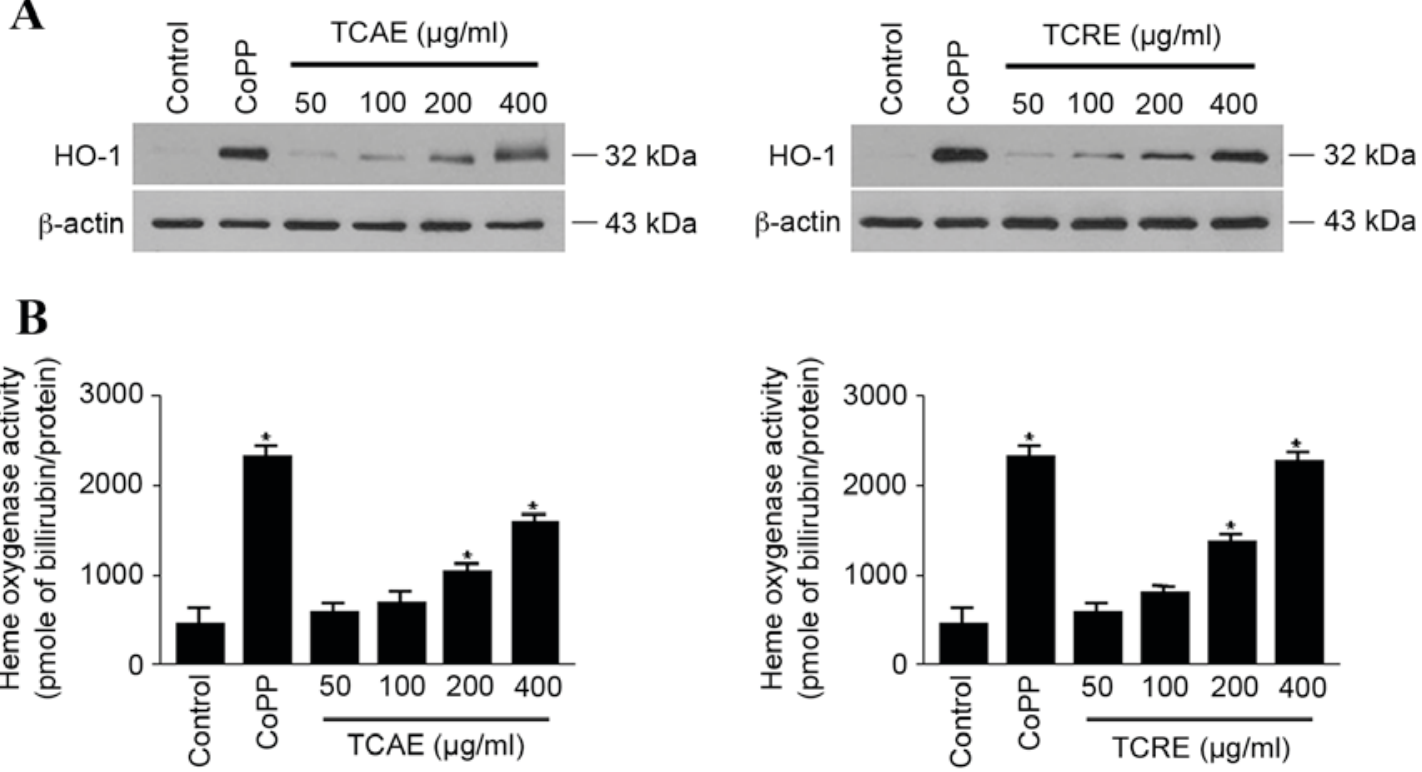

Figure 3. Effects of TCAE and TCRE on HO-1 expression and activity. HT22 cells were treated with 0, 50, 100, 200 or $400 \mu \mathrm{g} / \mathrm{ml}$ TCAE or TCRE extract for $12 \mathrm{~h}$. CoPP $(20 \mu \mathrm{M})$ treatment was used as a positive control. (A) HO-1 protein expression was analyzed by western blot (representative images). (B) HO-1 activity. "P $<0.05$ vs. control (untreated cells). TCAE, Taraxacum coreanum aerial extract; TCRE, T. coreanum root extract; HO-1, heme oxygenase-1; CoPP, cobalt protoporphyrin IX.

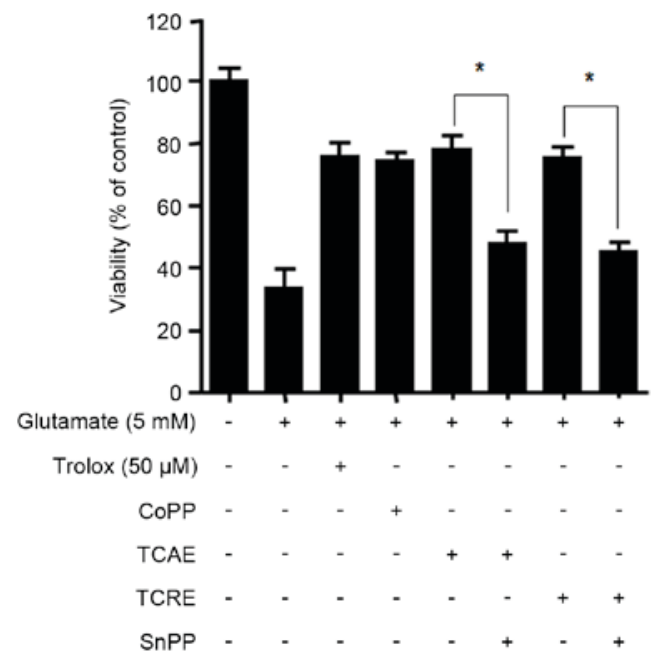

Figure 4. Effects of HO-1 inhibitor SnPP on TCAE- and TCRE-mediated cytoprotection. To test the effect of HO-1 inhibition, cells were pretreated with SnPP $(50 \mu \mathrm{M})$ prior to TCAE/TCRE-treatment. Cells were then incubated with either trolox (50 $\mu \mathrm{M}$, positive control), CoPP $(20 \mu \mathrm{M}$, positive control), TCAE $(400 \mu \mathrm{g} / \mathrm{ml})$ or TCRE $(400 \mu \mathrm{g} / \mathrm{ml})$. Cell damage was induced by treatment with $5 \mathrm{mM}$ glutamate in HT22 cells for $12 \mathrm{~h}$. Viability was measured by MTT assay. ${ }^{*} \mathrm{P}<0.05$, with comparisons indicated by brackets. HO-1, heme oxygenase-1; SnPP, tin protoporphyrin; TCAE, Taraxacum coreanum aerial extract; TCRE, T. coreanum root extract; CoPP, cobalt protoporphyrin IX.

competitive inhibitor of HO-1, significantly reduced the cytoprotective effects of TCAE and TCRE on glutamate-induced cell damage $(\mathrm{P}<0.05$; Fig. 4). The present data, therefore, suggested that the neuroprotective effects of TCAE and TCRE against oxidative stress are a result of their ability to induce HO-1 expression.

Effects of TCAE and TCRE on Nrf2 nuclear translocation. In order to examine whether Nrf2 is involved in the

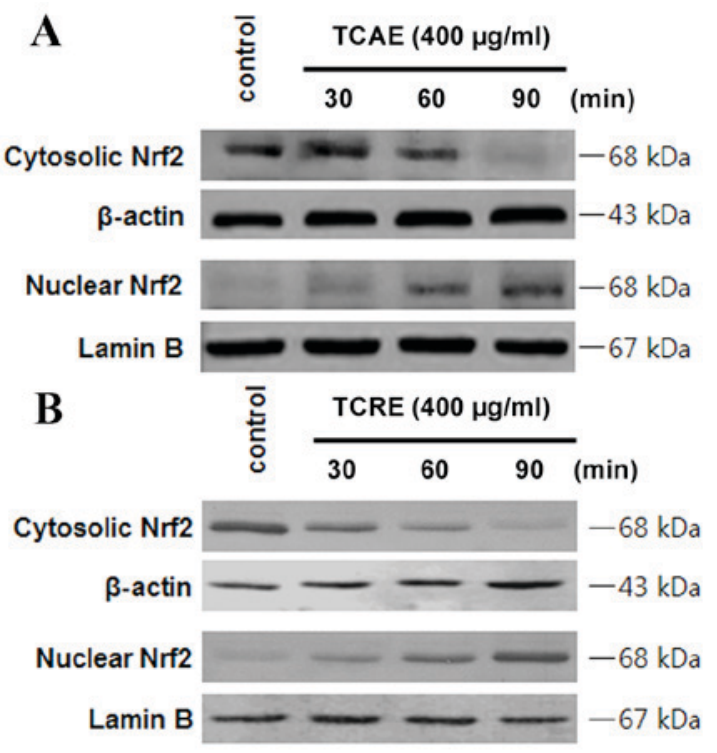

Figure 5. Effects of (A) TCAE and (B) TCRE on Nrf2 nuclear translocation. HT22 cells were either untreated (control) or incubated with $400 \mu \mathrm{g} / \mathrm{ml}$ TCAE or TCRE for 30,60 or $90 \mathrm{~min}$. The nuclear and cytosolic fractions were then separated and assayed for Nrf 2 expression by western blot. $\beta$-actin was used as an internal control for the cytoplasmic fraction, and lamin B for the nuclear fraction. TCAE, Taraxacum coreanum aerial extract; TCRE, T. coreanum root extract; Nrf2, nuclear factor erythroid 2 like 2.

extract-mediated HO-1 induction, the effect on Nrf2 nuclear translocation in HT22 cells treated with TCAE and TCRE was tested (Fig. 5). HT22 cells were incubated with $400 \mu \mathrm{g} / \mathrm{ml}$ TCAE (Fig. 5A) or TCRE (Fig. 5B) for 30, 60 and $90 \mathrm{~min}$, then Nrf2 protein levels in the nuclear and cytosolic fractions were examined by western blot analysis. The nuclear extracts of both TCAE and TCRE-treated cells exhibited a timedependent increase in Nrf2 levels compared with untreated 
control, while Nrf2 levels decreased in the cytoplasmic fraction of HT22 cells (Fig. 5).

\section{Discussion}

Taraxacum is a taxonomically complex genus of the family Asteraceae. Some botanists have classified the genus into 34 macrospecies, and 2000 microspecies, while other botanists only accept a total of $\sim 60$ species (16). Taraxacum coreanum Nakai is a dandelion native to Korea and is widely consumed as a vegetable and as a traditional medicine for inflammatory disorders in Korea. Although the whole plant of T. coreanum has been used in Korean traditional medicine, the present study aimed to evaluate the neuroprotective effects of its aerial and root parts separately. TCAE and TCRE ethanolic extracts were subjected to HPLC for evaluation of their chemical profile. As demonstrated in Fig. 1, the peaks of caffeic acid and ferulic acid were apparent in both extracts from their HPLC chromatograms. These results, therefore, provide insights into the chemical composition in the aerial and the root parts of T. coreanum.

Glutamate is one of the most important transmitters for brain function and is an important neural activator, however, excess glutamate can trigger neurodegenerative diseases (2). It is generally acknowledged that glutamate neurotoxicity is mediated by non-receptor-mediated oxidative stress and receptor-initiated excitotoxicity (2). To examine the neuroprotective effects of TCAE and TCRE, these extracts were added at non-toxic concentrations $(50-400 \mu \mathrm{g} / \mathrm{ml})$ in mouse hippocampal HT22 cells treated with glutamate. Both extracts significantly increased the survival of glutamate-treated HT22 cells (Fig. 2). HT22 cells lack glutamate receptors (5), suggesting that the protective effects of TCAE and TCRE are likely derived from their anti-oxidative properties rather than receptor-mediated signaling. In addition, caffeic acid and ferulic acid, which were used as internal standards in this study, did not exhibit any cytoprotective effects at their non-toxic concentrations against glutamate-induced HT22 cells (data not shown), suggesting that other components exist in TCAE and TCRE that are responsible for their neuroprotective activity.

$\mathrm{HO}-1$ is induced as a protective enzyme in response to diverse stimuli, therefore it may be suitable for the therapy of oxidative tissue damage (17). Therefore, the hypothesis that TCAE and TCRE could affect HO-1 expression was tested. The present study demonstrated that both extracts induced expression and activity of HO-1 in a concentration-dependent manner (Fig. 3). Furthermore, the neuroprotective activity of TCAE and TCRE were reversed by the HO-1 inhibitor SnPP (Fig. 4), suggesting that the protective properties of these extracts are mediated by HO-1.

HO-1 expression is associated with Nrf2 translocation (18). Under normal conditions, Nrf2 interacts with Kelch-like $\mathrm{ECH}$-associated protein 1 (Keap1) in the cytoplasm, but signals from electrophilic stimuli or increased reactive oxygen species production result in separation of the Nrf2-Keap1 complex, and Nrf2 translocation to the nucleus. Following nuclear translocation, Nrf 2 interacts with antioxidant response element sites in the promoter regions of specific target genes, initiating transcription of antioxidative genes, including GSH and HO-1 (19). The hypothesis that TCAE and TCRE may cause Nrf2 to translocate into the nucleus was, therefore, examined in HT22 cells. The results confirmed that Nrf2 levels were increased in the nuclear fractions of HT22 cells following TCAE and TCRE treatments, while Nrf2 levels decreased in the cytoplasm (Fig. 5), indicating increased translocation as a result of TCAE and TCRE treatment.

The present study revealed that $T$. coreanum, a native plant of Korea, confers a protective effect against glutamate-induced oxidative stress in HT22 cells. This cytoprotective effect of T. coreanum is mediated by induction of HO-1 expression, via Nrf2 nuclear translocation. Therefore, T. coreanum might serve as a potential therapeutic agent for preventing neurodegeneration and further studies are warranted for the isolation and characterization of its active substances.

\section{Acknowledgements}

The authors acknowledge the support from the Wonkwang University (Iksan, Korea) in 2015.

\section{References}

1. Uttara B, Singh AV, Zamboni P and Mahajan RT: Oxidative stress and neurodegenerative diseases: A review of upstream and downstream antioxidant therapeutic options. Curr Neuropharmacol 7: 65-74, 2009.

2. Schubert D and Piasecki D: Oxidative glutamate toxicity can be a component of the excitotoxicity cascade. J Neurosci 21: 7455 7462, 2001.

3. Davis JB and Maher P: Protein kinase C activation inhibits glutamate-induced cytotoxicity in a neuronal cell lines. Brain Res 652: 169-173, 1994.

4. Murphy TH, Miyamoto M, Sastre A, Schnaar RL and Coyle JT: Glutamate toxicity in a neuronal cell line involves inhibition of cystine transport leading to oxidative stress. Neuron 2: 1547$1558,1989$.

5. Rössler OG, Bauer I, Chung HY and Thiel G: Glutamate-induced cell death of immortalized murine hippocampal neurons: Neuroprotective activity of heme oxygenase-1, heat shock protein 70 and sodium selenite. Neurosci Lett 362: 253-257, 2004.

6. Cabell L, Ferguson C, Luginbill D, Kern M, Weingart A and Audesirk G: Differential induction of heme oxygenase and other stress proteins in cultured hippocampal astrocytes and neurons by inorganic lead. Toxicol Appl Pharmacol 198: 49-60, 2004.

7. Satoh T, Baba M, Nakatsuka D, Ishikawa Y, Aburatani H, Furuta K, Ishikawa T, Hatanaka H, Suzuki M and Watanabe Y: Role of heme oxygenase-1 protein in the neuroprotective effects of cyclopentenone prostaglandin derivatives under oxidative stress. Eur J Neurosci 17: 2249-2255, 2003.

8. Itoh K, Chiba T, Takahashi S, Ishii T, Igarashi K, Katoh Y, Oyake T, Hayashi N, Satoh K, Hatayama I, et al: An Nrf2/small Maf heterodimer mediates the induction of phase II detoxifying enzyme genes through antioxidant response elements. Biochem Biophys Res Commun 236: 313-322, 1997.

9. Ishii T, Itoh K, Takahashi S, Sato H, Yanagawa T, Katoh Y, Bannai $\mathrm{S}$ and Yamamoto M: Transcription factor Nrf2 coordinately regulates a group of oxidative stress-inducible genes in macrophages. J Biol Chem 275: 16023-16029, 2000.

10. Choi JH, Shin KM, Kim NY, Hong JP, Lee YS, Kim HJ, Park HJ and Lee KT: Taraxinic acid, a hydrolysate of sesquiterpene lactone glycoside from the Taraxacum coreanum NAKAI, induces the differentiation of human acute promyelocytic leukemia HL-60 cells. Biol Pharm Bull 25: 1446-1450, 2002.

11. Lee MH, Kang H, Lee K, Yang G, Ham I, Bu Y, Kim H and Choi HY: The aerial part of Taraxacum coreanum extract has an anti-inflammatory effect on peritoneal macrophages in vitro and increases survival in a mouse model of septic shock. J Ethnopharmacol 146: 1-8, 2013.

12. Lee DS, Ko W, Kim DC, Kim YC and Jeong GS: Cudarflavone $\mathrm{B}$ provides neuroprotection against glutamate-induced mouse hippocampal HT22 cell damage through the Nrf2 and PI3K/Akt signaling pathways. Molecules 19: 10818-10831, 2014. 
13. Motterlini R, Foresti R, Intaglietta $\mathrm{M}$ and Winslow $\mathrm{RM}$ : NO-mediated activation of heme oxygenase: Endogenous cytoprotection against oxidative stress to endothelium. Am J Physiol 270: H107-H114, 1996.

14. Li XP, Yu J, Luo JY, Li HS, Han FJ, Chen XG and Hu ZD Simultaneous determination of chlorogenic acid, caffeic acid, ferulic acid, protocatechuic acid and protocatechuic aldehyde in Chinese herbal preparation by RP-HPLC. Chem Pharm Bull 52: 1251-1254, 2004.

15. Ling Y, Zhang Y, Cai S, Xiao Y, Cai S and Zheng J: Chemical constituents of Taraxacum sinicum Kitag. Zhongguo Zhing Yao Za Zhi 23: 232-234, 1998 (In Chinese).

16. Richards AJ: Eutriploid facultative agamospermy in Taraxacum. New Phytologist 69: 761-774, 1970.
17. Farombi EO and Surh YJ: Heme oxygenase-1 as a potential therapeutic target for hepatoprotection. J Biochem Mol Biol 39: 479-491, 2006

18. Moi P, Chan K, Asunis I, Cao A and Kan YW: Isolation of NF-E2-related factor 2 (Nrf2), a NF-E2-like basic leucine zipper transcriptional activator that binds to the tandem NF-E2/AP1 repeat of the beta-globin locus control region. Proc Natl Acad Sci USA 91: 9926-9930, 1994.

19. Qiang W, Cahill JM, Liu J, Kuang X, Liu N, Scofield VL, Voorhees JR, Reid AJ, Yan M, Lynn WS and Wong PK: Activation of transcription factor Nrf-2 and its downstream targets in response to moloney murine leukemia virus ts1-induced thiol depletion and oxidative stress in astrocytes. J Virol 78: 11926-11938, 2004. 